\title{
Bandwidth optimization for filter-based fatigue index in different inter-electrode distances
}

\author{
Jungyoon Kim, Jongsang Son and Youngho Kim* \\ Department of Biomedical Engineering and Institute for Convergence Study of Bio-Medical Wellness, \\ Yonsei University (Wonju Campus), Maeji-ri, Heungeop-myeon, Wonju-si, Gangwon-do, 220-710 \\ Republic of Korea
}

\begin{abstract}
In this study, the bandwidth of the filter-based fatigue index was determined by the comparison of optimized cutoff frequencies in different inter-electrode distances. Sixty-one subjects participated in isometric knee extension, isotonic ankle dorsiflexion, and isotonic elbow extension exercises. Electromyography (EMG) signals were obtained from right rectus femoris, triceps brachii, and tibialis anterior muscles during exercises. The filter-based fatigue index was compared with mean root-mean-square values, median frequency, Dimitrov spectral index, and Gonzalez-Izal wavelet index. Optimized cutoff frequencies of the high-pass filter for three different exercises and three different inter-electrode distances were about 350 $\mathrm{Hz}$. Results from this study support that around $350 \mathrm{~Hz}$ high-pass filter could be useful to determine cut-off frequency for fatigue prediction in general purposes.
\end{abstract}

Keywords: Electromyography, fatigue, filter, bandwidth optimization, inter-electrode distance

\section{Introduction}

Muscle fatigue has been defined as "a response that is less than the expected or anticipated contractile response, for a given stimulation" [1]. Hard physical exercise or prolonged physical work involving $30 \%$ to $40 \%$ of maximal aerobic capacity can induce muscle fatigue [2]. Muscle fatigue is a hindrance during exercise, as it causes dysfunction [3]. In transportation industry, frequent and repetitive sub-maximal contraction and incorrect posture lead to many disorders of muscles, tendons and nerves [4]. Repetition of the same task and the prolonged abnormal posture contributes to upper limbs and neck disorders [5]. In sports, muscle fatigue plays an important role in prolonged contractile activityinduced skeletal muscle injuries [6]. In the transportation industry, fatigue-related accidents are major killers, accounting for $25 \%$ of vehicle accidents [7]. Therefore, it is very important to measure muscle fatigue.

\footnotetext{
${ }^{*}$ Corresponding author: Youngho Kim, Department of Biomedical Engineering and Institute for Convergence Study of Bio-Medical Wellness, Yonsei University (Wonju Campus), Maeji-ri, Heungeop-myeon, Wonju-si, Gangwon-do, 220-710 Republic of Korea. Tel.: +82-33-760-2492; Fax: +82-33-760-2806; E-mail: younghokim@yonsei.ac.kr.
}

0959-2989/14/\$27.50 @ 2014 - IOS Press and the authors. 
Mean and median frequencies ( $\mathrm{F}_{\text {mean }}$ and $\mathrm{F}_{\text {med }}$, respectively) are commonly used to estimate fatigue. Using the electromyography (EMG), $F_{\text {mean }}$ and $F_{\text {med }}$ detect that power spectrum shifts to the lower frequency range and muscle fiber conduction velocity (MFCV) decreases during fatigue [8]. However, they have a relatively low sensitivity under dynamic exercise conditions [9].To cope with this sensitivity problem, various attempts have been performed [10-12]. Karlsson et al. [10] tested the short-time Fourier transform, the Wigner-Ville distribution, the Choi-Williams distribution and the continuous wavelet transform (CWT) during isokinetic knee extension exercises, and concluded that the estimates provided by the CWT show the best accuracy compared with the other methods. Dimitrov et al. [11] proposed a high-sensitivity spectral index, the ratio of the spectral moments of order -1 to the spectral moment of order five using the fast Fourier transform. Their index $\mathrm{FI}_{n s m 5}$ was eight times as large as the initial value during cycling exercises. Gonzalez-Izal et al. [12] combined the ratio of different spectral moments and the discrete wavelet transforms (DWT) and tested it during leg press exercises. Their index WIRE51 showed a good correlation with peak power output and the robustness against noise. However, large amounts of computational load and time-frequency resolution were the drawbacks of those methods [13].

Kim et al. [13] proposed a filter-based fatigue index defined by the ratio of high- and low-frequency contents of EMG signals. It has the same order as time resolution and less computational load. However, there was no criterion for bandwidth selection. The cut-off frequencies of the high-pass filter were also optimized to maximize the correlation coefficient between peak power and their filter-based fatigue index $\left(\mathrm{FI}_{\text {hlrOPT }}\right)$ [14]. Their index $\mathrm{FI}_{\text {hlropt }}$ showed better correlation with peak power than previous fatigue indices (RMS, $\mathrm{F}_{\text {med }}, \mathrm{FI}_{\mathrm{nsm} 5}$, WIRE51). However, they assessed only isotonic knee extension exercise and tested it only for $1.8 \mathrm{~cm}$ inter-electrode distance.

In this study, the bandwidth of the filter-based fatigue index was determined by the comparison of optimized cut-off frequencies in different inter-electrode distances, assuming that optimized cut-off frequencies in various inter-electrode distances would be invariant. The filter-based fatigue index was also compared with the conventional median frequency, the spectral parameter proposed by Dimitrov [11], and the wavelet index proposed by Gonzalez-Izal [12].

\section{Subjects and methods}

\subsection{Subjects}

Sixty-one healthy male adults (age: $24.7 \pm 5.2$ years, height: $172.1 \pm 7.8 \mathrm{~cm}$, weight: $79.3 \pm 11.4 \mathrm{~kg}$ ) were recruited in this study. All subjects had no history of limb disorders. They performed isometric knee extension exercises, isotonic elbow extension and isotonic ankle dorsiflexion. Subjects were informed about the purpose of the study and the experimental procedure. They provided the written informed consents before participating in the study. The Yonsei University Research Ethics Committee reviewed and approved the experimental design of the present study (1041849-201405-BM-022-02).

\subsection{Experimental methods}

A Biodex System III Dynamometer (Biodex Medical Systems, NY, USA) was used to measure joint torque during flexion/extension exercises. Before the test started, each subject practiced at submaximal contraction levels. Three kinds of exercises were selected to validate the filter-based fatigue index. 


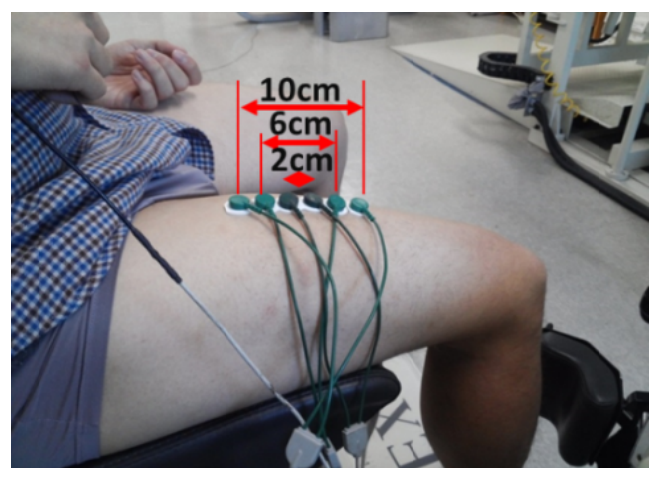

Fig. 1. Different inter-electrode distances $(2 \mathrm{~cm}, 6 \mathrm{~cm}$ and $10 \mathrm{~cm})$.

In isotonic exercises, each subject performed several maximal voluntary contractions (MVC) to determine the 10 repetition maximum (10RM) load for fatigue exercises. The fatigue exercise consisted of five sets of ten joint movements with two minutes between-set rest interval. Subjects were asked to perform joint movements with their own maximum efforts during fatigue exercises. In elbow extension exercise, range of motion (ROM) was from $100^{\circ}$ to $40^{\circ}$ and the determined 10RM was 64.7 $\pm 3.8 \%$ of the maximum torque. In ankle dorsiflexion exercise, ROM was from $-25^{\circ}$ to $5^{\circ}$ and the determined $10 \mathrm{RM}$ was $60.3 \pm 5.4 \%$ of the maximum torque.

In isometric knee extension exercises, EMG signals and joint torques were measured at the knee joint angle of $90^{\circ}$ during MVC. The experimental protocol was based on the maximal isometric knee extension until exhaustion.

\subsection{Data collection}

An EMG measurement system (MyoSystem 1200, Noraxon, AZ, USA) and bipolar surface EMG (sEMG) electrodes (Ag-AgCl, $14 \mathrm{~mm}$ diameter; Noraxon, AZ, USA) were used to measure EMG signals from right rectus femoris (RF), triceps brachii (TC) and tibialis anterior (TA) muscles during exercises. The inter-electrode distance was set to $2 \mathrm{~cm}, 6 \mathrm{~cm}$ and $10 \mathrm{~cm}$ during isotonic elbow extension, isotonic ankle dorsiflexion and isometric knee extension, respectively (see Figure 1). Based on the Surface Electromyography for the Non-Invasive Assessment of Muscles (SENIAM) recommendations [15], EMG electrode pairs were placed on the midway of the muscle. The shaved skin at each electrode site was carefully abraded and cleaned with alcohol. Isokinetic dynamometer was used to measure joint angle, angular velocity and torque. All signals were simultaneously recorded at a sampling rate of $1 \mathrm{kHz}$.

\subsection{Data analysis}

Data was analyzed using MATLAB software (The Math Works Inc., Natick, MA, USA). During isotonic exercises, all data was divided into fifty segments, and a segment was defined as one exercise repetition. In isometric exercises, full period (from contraction start to exhaustion) of exercise was divided into fifty segments.

Maximum torque was calculated as the mean value over a one-second period with relatively torqueplateau levels of each MVC exercise. In isotonic exercises, powers of each segment were calculated 
by the product of joint torque and angular velocity. The following EMG parameters were used to estimate muscle fatigue:

1. Mean root-mean-square (RMS) using a 50-ms window.

2. Median frequency $\left(\mathrm{F}_{\mathrm{med}}\right)$

3. Spectral parameter $\left(\mathrm{FI}_{\mathrm{nsm} 5}\right)[11]$

4. Wavelet index(WIRE51) [12]

5. Filter-based fatigue index [14]

The filter-based fatigue index was calculated by:

$$
\mathrm{FI}_{\text {hlropt }}=\int_{\text {satr }}^{\text {end }} \frac{\mathrm{LE}_{\text {OPT HzHPF }}(\mathrm{t})}{\mathrm{LE}_{200 \mathrm{HZLPF}}(\mathrm{t})} \mathrm{dt} /\left(\mathrm{t}_{\text {end }}-\mathrm{t}_{\text {start }}\right)
$$

where OPT was the optimized cut-off frequency of the high-pass filter, $t_{\text {start }}$ and $t_{\text {end }}$ were the start and end time for each segment, respectively. $\mathrm{LE}_{200 \mathrm{HzLPF}}$ and $\mathrm{LE}_{\mathrm{OPTHzHPF}}$ were linear-enveloped signals of the $200 \mathrm{~Hz}$ low-pass filtered sEMG and those of the OPT Hz high-pass filtered sEMG, respectively. The linear-enveloped signal was the 2nd-order Butterworth low-pass filter (3 Hz cut-off frequency) after full rectification of EMG signal. The cut-off frequency of high-pass filter was optimized using the interior-point algorithm [16] to maximize the correlation coefficient between $\mathrm{FI}_{\mathrm{hl} \text { IOPT }}$ and peak powers or joint torques. The cut-off frequency of the low-pass filter $(200 \mathrm{~Hz})$ was determined to retain more than $95 \%$ of the power of raw EMG signals [14].

\subsection{Statistical analysis}

All variables were normalized by the mean value of the first two segments. In cases where the percentage changes did not follow a normal distribution $\left(\mathrm{FI}_{\mathrm{nsm}}\right.$ and WIRE), the corresponding variables were log-transformed [12]. One-way analysis of variance (ANOVA) was used to compare optimized cut-off frequencies for three different exercise methods. Significant differences in the correlation coefficients for different inter-electrode distances were also calculated by ANOVA analysis. Tukey's studentized range test was performed to identify homogeneous subsets of means that are identical each other. The relationship between peak powers (for isotonic and isokinetic exercises) or joint torques (for isometric exercises) and different EMG indices were calculated using Pearson's correlation coefficients. Differences between mean determination coefficients for $\mathrm{FI}_{\text {hrlOPT }}$ and the other EMG indices were calculated by the unpaired $t$-test.

\section{Results}

\subsection{Influence of various inter-electrode distances}

Figure 2 shows EMG amplitude and $\mathrm{F}_{\text {med }}$ in various inter-electrode distances during isotonic elbow extension, isotonic ankle dorsiflexion and isometric knee extension. EMG amplitude increased with the increasing inter-electrode distance except isotonic ankle dorsiflexion in $10 \mathrm{~cm}$ inter-electrode distance. $\mathrm{F}_{\text {med }}$ decreased with the increasing inter-electrode distance in three different exercises. 


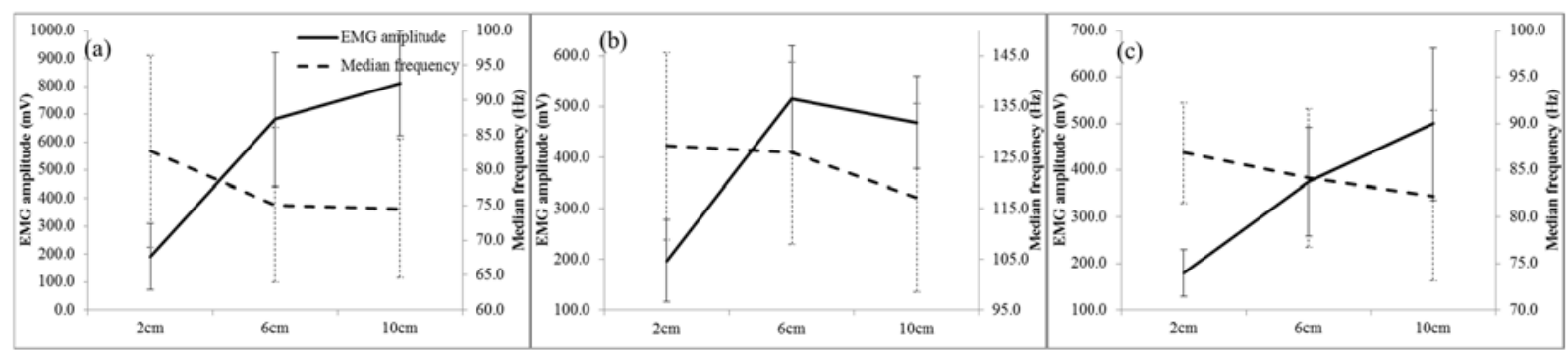

Fig. 2. EMG amplitude and $\mathrm{F}_{\mathrm{med}}$ in various inter-electrode distances during (a) isotonic elbow extension, (b) isotonic ankle dorsiflexion and (c) isometric knee extension.

\subsection{Bandwidth optimization in various inter-electrode distances}

Table 1 shows the optimized cut-off frequencies and correlation coefficients between $\mathrm{FI}_{\mathrm{hl} O \mathrm{OPT}}$ and biomechanical fatigues in different inter-electrode distances during isotonic elbow extension, isotonic ankle dorsiflexion and isometric knee extension. Optimized cut-off frequencies of all muscles and all inter-electrodes were identified within the same subset $(\mathrm{p}=0.811)$. Correlation coefficients from the same muscle were classified into the same group $(p=0.823$ for TC, $p=0.998$ for TA and $p=1.00$ for $\mathrm{RF})$.

Table 1

Optimized cut-off frequencies and correlation coefficients between $\mathrm{FI}_{\mathrm{hl} \text { rOPT }}$ and peak powers or joint torques in different inter-electrode distances

\begin{tabular}{llll}
\hline & Inter-electrode distance $(\mathrm{cm})$ & Optimized cut-off frequency $(\mathrm{Hz})$ & Correlation coefficient \\
\hline \multirow{2}{*}{$\mathrm{TC}$} & 2 & $357.5 \pm 46.0$ & $0.71 \pm 0.12$ \\
& 6 & $351.4 \pm 65.4$ & $0.69 \pm 0.14$ \\
$\mathrm{TA}$ & 10 & $357.6 \pm 54.6$ & $0.75 \pm 0.08$ \\
& 2 & $355.3 \pm 51.3$ & $0.58 \pm 0.13$ \\
& 6 & $348.1 \pm 38.2$ & $0.58 \pm 0.16$ \\
$\mathrm{RF}$ (isometric) & 10 & $345.1 \pm 44.7$ & $0.60 \pm 0.12$ \\
& 2 & $357.4 \pm 45.4$ & $0.92 \pm 0.05$ \\
& 10 & $361.4 \pm 61.2$ & $0.92 \pm 0.04$ \\
\hline
\end{tabular}

Note: Boxes indicate the same subset.

Table 2

Determination coefficients between EMG parameters and peak powers or joint torques in $2 \mathrm{~cm}$ inter-electrode distance

\begin{tabular}{llllll}
\hline & $\mathrm{RMS}$ & $\mathrm{F}_{\text {med }}$ & $\mathrm{FI}_{\mathrm{nsm} 5}$ & WIRE51 & $\mathrm{FI}_{\mathrm{hlrOPT}}$ \\
$\begin{array}{l}\text { Isotonic } \\
\text { elbow extension }\end{array}$ & $0.28 \pm 0.18^{*, \dagger}$ & $0.36 \pm 0.15^{*}$ & $0.33 \pm 0.22^{*, \dagger}$ & $0.31 \pm 0.08^{*, \dagger}$ & $0.51 \pm 0.16$ \\
$\begin{array}{l}\text { Isotonic } \\
\text { ankle dorsiflexion }\end{array}$ & $0.27 \pm 0.16^{*, \dagger}$ & $0.23 \pm 0.18^{*}$ & $0.32 \pm 0.17^{\dagger}$ & $0.14 \pm 0.13^{*, \dagger}$ & $0.35 \pm 0.15$ \\
$\begin{array}{l}\text { Isometric } \\
\text { knee extension }\end{array}$ & $0.60 \pm 0.26^{*}$ & $0.63 \pm 0.16^{*}$ & $0.47 \pm 0.12^{*, \dagger}$ & $0.58 \pm 0.14^{*, \dagger}$ & $0.86 \pm 0.08$ \\
\hline
\end{tabular}

Note: ${ }^{*} p<0.05$. Differences from $\mathrm{FI}_{\mathrm{hlrOPT}}{ }^{\dagger}$ negative correlation. 


\subsection{Determination coefficients between EMG parameters and peak powers or joint torques}

Table 2 shows the determination coefficients between EMG parameters and peak powers or joint torques for three different exercises in $2 \mathrm{~cm}$ inter-electrode distance. In all exercises, $\mathrm{FI}_{\text {hlroPT }}$ showed significantly higher correlation with peak power or joint torque than the other fatigue indices. $\mathrm{FI}_{\mathrm{nsm} 5}$ and WIRE51 showed the negative correlation, but $\mathrm{F}_{\text {med }}$ and $\mathrm{FI}_{\text {hlrOp }}$ showed the positive correlation with peak power or joint torque. RMS values showed the negative correlation with peak powers during isotonic exercises, but showed the positive correlation with joint torques during isometric exercises.

\section{Discussion}

This study assumed that optimized cut-off frequencies would be invariant for different interelectrode distances. EMG amplitude increased with the increasing inter-electrode distance due to the larger detection volume [17]. EMG spectrum was also affected by inter-electrode distance. EMG power spectrum shifted to the lower frequency due to the lengthening effect of MUAP with the increasing inter-electrode distance [18]. Three different inter-electrode distances were used to verify these effects. As the inter-electrode distance increased, EMG amplitude increased but $F_{\text {med }}$ decreased $[17,18]$. However, optimized cut-off frequencies in three different exercises and in three different inter-electrode distances were classified into the same group. This supports our assumption that the optimized cut-off frequencies in various inter-electrode distances are constant, and also supports the previous result that the $\mathrm{FI}_{\mathrm{hlrOPT}}$ reflects firing rate of fast twitch muscle fibers [14]. Correlations with peak power or joint torque from the same muscle were also identified as the same subset. This indicates that the normalized $\mathrm{FI}_{\mathrm{hl} \text { IOPT }}$ was not affected by the inter-electrode distance.

Three muscles and two types of exercises were included in this study. The average fiber type distribution for RF muscle was 52\% type I and 48\% type II [19], while that for TC muscle was $43 \%$ type I and $57 \%$ type II [20], and for TA muscle was $77 \%$ type I and $23 \%$ type II [21]. During isometric exercises, joint torque and joint angular velocity decreased naturally during isotonic exercises. In this study, RMS value showed the negative correlation with peak power during isotonic exercises and the positive correlation with joint torque during isometric exercise. However, optimized cut-off frequencies were identified as the same group, and $\mathrm{FI}_{\text {hlropt }}$ showed the best correlation with peak power or joint torque. Similar to the previous studies $[13,14]$, an optimized cut-off frequency is found to be about $350 \mathrm{~Hz}$ in this study. This result supports that the filter-based fatigue index could assess the muscle fatigue in different muscles during different exercise with $350 \mathrm{~Hz}$ high-pass filter.

Correlation coefficients in TA muscle were smaller than other muscles, because the load of isokinetic dynamometer was relatively larger than other exercises. Correlation coefficients in RF muscle were greater than other muscles because isometric exercise was the best method to estimate fatigue.

Although ratios of the EMG power components in high- and low-frequency contents showed good accuracy of fatigue estimation, high- and low-frequency bandwidth is still difficult to be selected $[13,22-$ 24]. To overcome this limitation, Kim et al. [14] determined the cut-off frequencies of the high-pass filter by maximizing the correlation coefficient between peak power and their filter-based fatigue index. The determined frequencies were consistent (approximately $350 \mathrm{~Hz}$ ), showing 353 and $349 \mathrm{~Hz}$ in RF and vastii muscles, respectively. However, they did not confirm whether it can be used in other types of exercises or different muscles. In this study, it was observed that $\mathrm{FI}_{\mathrm{hlropT}}$ showed the significantly higher correlation with peak power or joint torque than other fatigue indices in all conditions. This suggested 
that $\mathrm{FI}_{\mathrm{hl} \text { Irop }}$ had the best accuracy to estimate muscle fatigue. The present results support that around $350 \mathrm{~Hz}$ high-pass filter could be useful to estimate muscle fatigue in general purposes.

\section{Conclusion}

In this study, the bandwidth of the filter-based fatigue index was determined by the comparison of optimized cut-off frequencies in different inter-electrode distances. Results showed that optimized cutoff frequencies of three exercises and three inter-electrode distances are consistent, implying that 350 $\mathrm{Hz}$ HPF could be a general solution for the $\mathrm{FI}_{\text {hlrOPT. }}$. $\mathrm{FI}_{\text {hlrOPT }}$ shows the best correlation with peak power or joint torque in all conditions. This information could be useful for muscle fatigue assessment.

\section{Acknowledgement}

This research was financially supported by the Ministry of Education (MOE) and National Research Foundation of Korea (NRF) through the Human Resource Training Project for Regional Innovation (No. 2013H1B8A2032194), and a grant from the Korean Health Technology R \& D Project, Ministry of Health \& Welfare, Republic of Korea (HI10C2017(A102062)).

\section{References}

[1] B.R. MacIntosh and D.E. Rassier, What is fatigue? Can. J. Appl. Physiol. 27 (2002), 42-55.

[2] P. Astrand and K. Rodhal, Textbook of Work Physiology, Physiological Bases of Exercise, McGraw-Hill Book Company, NewYork, 1986.

[3] H. Ishii and Y. Nichida, Effect of lactate accumulation during exercise-induced muscle fatigue on the sensorimotor cortex, J. Phys. Ther. Sci. 25 (2013), 1637-1642.

[4] Work-related Musculoskeletal Disorders (WMSDs), available at: http://www.ccohs.ca/oshanswers/diseases/rmirsi.html, January 8, 2014.

[5] K. Walker-Bone and C. Cooper, Hard work never hurt anyone: or did it? A review of occupational associations with soft tissue musculoskeletal disorders of the neck and upper limb, Annals of Rheumatic Diseases 64 (2005), 1391-1396.

[6] M. Hägglund, M. Waldén and J. Ekstrand, Risk factors for lower extremity muscle injury in professional soccer the UEFA injury study, Am. J. Sports. Med. 41 (2013), 327-335.

[7] E. Sung, B. Min, S. Kim and C. Kim, Effects of oxygen concentrations on driver fatigue during simulated driving, Appl. Ergon. 36 (2005), 25-31.

[8] K. Masuda, T. Masuda, T. Sadoyama, M. Inaki and S. Katsuta, Changes in surface EMG parameters during static and dynamic fatiguing contractions, J. Electromyogr. Kinesiol. 9 (1999), 39-46.

[9] D. Farina, R. Merletti and R.M. Enoka, The extraction of neural strategies from the surface EMG, J. Appl. Physiol. 96 (2004), 1486-1495.

[10] S. Karlsson, J. Yu and M. Akay, Time-frequency analysis of myoelectric signals during dynamic contractions: A comparative study, IEEE Trans. Biomed. Eng. 47 (2000), 228-238.

[11] G.V. Dimitrov, T.I. Arabadzhiev, K.N. Mileva, J.L. Bowtell, N. Crichton and N.A. Dimitrova, Muscle fatigue during dynamic contractions assessed by new spectral indices, Med. Sci. Sports Exerc. 38 (2006), 1971-1979.

[12] M. Gonzalez-Izal, I. Rodríguez-Carreño, A. Malanda, F. Mallor, I. Navarro-Amezqueta, E.M, Gorostiaga and M. Izquierdo, sEMG wavelet-based indices predicts muscle power loss during dynamic contractions, J. Electromyogr. Kinesiol. 20 (2010), 1097-1106.

[13] J. Kim, S. Park, S. Ahn and Y. Kim, A novel approach of defining fatigue indices with sEMG power during isotonic contractions, J. Precis. Eng. Manuf. 13 (2010), 977-983.

[14] J. Kim, S. Kim, S. Park, S. Ahn, Y. Lee and Y. Kim, Bandwidth optimization of the fatigue index to estimate muscle fatigue during dynamic contractions, J. Precis. Eng. Manuf. 14 (2013), 1185-1191. 
[15] B. Freriks, C. Disselhorst-Klug, H.J. Hermens and G. Rau, Sensors and sensor placement procedures used in the European labs, in: SENIAM 5: The State of the Art on Sensors and Sensor Placement Procedures for Surface Electromyography: A Proposal for Sensor Placement Procedures, Roessingh Research and Development, Enschede, 1997, pp. 107113.

[16] M.H. Wright, The interior-point revolution in optimization: History, recent developments, and lasting consequences, Bull. Am. Math. Soc. 42 (2005), 39-56.

[17] T. Castroflorio, D. Farina, A. Botin, M.G. Piancino, P. Bracco and R. Merletti, Surface EMG of the jaw elevator muscles: Effect of electrode locations and inter-electrode distance, J. Oral. Rehabil. 32 (2005), 411-417.

[18] M.H. Malek, T.J. Houch, J.W. Coburn, J.P. Weir, R.J. Schmidt and T.W. Beck, The effects of inter-electrode distance on electromyographic amplitude, Journal of Neuroscience Methods 151 (2006), 139-147.

[19] R. Scelsi, Skeletal muscle pathology after spinal cord injury, our 20-year experience and results on skeletal muscle changes in paraplegics, related to functional rehabilitation, Basic Appl. Myol. 11 (2001), 75-86.

[20] P.G. Schantz, R. Billeter, J. Henriksson and E. Jansson, Training-induced increase in myofibrillar ATPase intermediate fibres in human skeletal muscle, Muscle Nerve 5 (1982), 628-636.

[21] F. Jakobsson, K. Borg and L. Edstrom, Fibre-type composition, structure and cytoskeletal protein location of fibers in anterior tibial muscle, Acta Neuropathol. 80 (1990), 459-468.

[22] J. Moxham, R.H.T. Edwards, M. Aubier, A. De Troyer, G. Farkas, P.T. Macklem and C. Roussos, Changes in EMG power spectrum (high-to-low ratio) with force fatigue in humans, J. Appl. Physiol. 53 (1982), 1094-1099.

[23] F. Bellemare and A. Grassino, Evaluation of human diaphragm fatigue, J. Appl. Physiol. 53 (1982), 1196-1206.

[24] K. Lee, K. Shin, H. Kim and J. Mun, Estimating muscle fatigue of the biceps brachii using high to low band ratio in EMG during isotonic exercise, Int. J. Precis. Eng. Manuf. 10 (2009), 147-153. 